\title{
Spatially Resolved Triboemission Measurements
}

J. Le Rouzic, T. Reddyhoff

Department of Mechanical Engineering, Imperial College London, London SW7 2AZ, United Kingdom

Corresponding author: Julian Le Rouzic

$+44(0) 2075947236$

j.le-rouzic@imperial.ac.uk

Keywords: Triboemission - Aluminium - Unlubricated wear - Wear mechanisms

\begin{abstract}
Electron emission that occurs when surfaces undergo wear has been studied since the 1950s, and is the subject of continued speculation due to its possibly crucial role in the formation of boundary lubricating films and the degradation of hard drive lubricants. Despite this, the mechanisms by which this type of emission occurs are poorly understood. In order to shed light on this phenomenon, we report for the first time how friction stimulated electron emission can be visualised, by scratching an aluminium oxide surface, positioned $10 \mathrm{~mm}$ from a microchannel plate in a high vacuum. The microchannel plate is coupled to a phosphor-screen displaying the spatial distribution of electrons that are emitted in individual bursts as wear is accruing. The probability density of this emission has been calculated and a Shannon spectral entropy of 0.11 for a 10 ms time window, suggesting partially deterministic mechanisms are responsible. These emission maps help to elucidate the obscure mechanisms that cause emission, in a way that has not been possible with previous spatially averaged measurements. For example, linear distributions support the theory that electron emission arises due to the formation of surface cracks. In addition to this, as the contact moves over the specimen surface, certain locations show an increased propensity to emit, which further suggests the presence of localised defects. Furthermore, in certain cases sustained after-emission is observed from localised defects exiting the contact. These results highlight the possibility of using the technique to monitor very low levels of wear and even image crack formation in real time.
\end{abstract}

\section{Introduction}

Triboemission is the collective term for friction stimulated emission of particles (electrons, ions, neutral particles, photons) that occurs under the severe conditions at the interface between sliding bodies [1]. This type of particle emission is distinct from emission due to other external stimulation, for example by photons (photoemission [2]) or by heat (thermoemission [3]).

The mechanisms that give rise to triboemission are as yet poorly understood. However, it is believed to initiate the chemical reactions that lead to the formation of surface films in boundary lubrication [4, 5]. For example, widely used zinc dialkyldithophosphate (ZDDP) additives produce boundary films by a mechanism that cannot be driven by contact temperatures and pressure alone [6]. In addition to this, tribolemission has been blamed for the 
degradation of computer hard drive lubricants [7] and has also been linked to flash lights during the fracture of rocks associated in earthquakes [8] and volcanic eruptions [9].

In order to understand the role of emitted particles in these applications so that boundary lubrication can be enhanced and lubricant degradation prevented - it is necessary to study the mechanisms by which emission occurs. For this reason, triboemission has been the focus of significant research efforts for over half a century [10].

The first significant triboemission measurements were made by Nakayama et al. during scratch tests by a diamond tip. To achieve this, they used a low-voltage biased collector positioned close to the contact that registered bursts of the electrical current due to the emission of negatively and positively particles [11], while photon emission was measured using a photomultiplier [12]. Their results showed that insulator materials produced significantly higher levels of emission when scratched than metals. Kim et al. investigated the emission of electrons using channel electron multipliers (CEMs) and photons using photomultipliers, during abrasion of MgO samples under vacuum. They also measured the kinetic energy of the electrons using a retarding grid [13]. In these studies, photon emission was attributed mainly to deformation and electrons emission to fracture. Molina et al. investigated the triboemission of negatively charged particles under vacuum for a wide range of materials (alumina, sapphire, silicon nitride and semiconductors) using a CEM in pulse counting mode [14-16]. The results from Nakayama et al. and Molina et al. suggested that triboemission is related to material hardness [14,17]. Emission is also correlated strongly to the wear of the surface, highlighted by the observation that repeated scratches along a single track results in a decrease of emission with time, due to decreasing wear [14].

From these investigations, it is generally concluded that triboemission of electrons is related to surface fracture during sliding contact. More specifically, Dickinson et al. [18] put forward a charge separation model, shown in Figure 1. Here, an imbalance of charge is created between opposite faces of a crack as it opens. Due to the small width of the crack, this electric field has a large gradient, which encourages electrons to leave the negatively charged crack face. Due to thermal vibrations, a portion of these electrons do not reach the opposing face and are instead emitted into the surrounding environment. This model however, does not explain why the energy of the tribo-emitted electrons can be lower than the work function of the sample [14]. To account for these low energy electrons, Dickinson et al. suggested that the work function of the surface could be locally decreased by small defects in the highly strained areas [18].

Since triboemission measurements typically contain no spatial information, researchers have tried to gain insights into the emission mechanisms by analysing the temporal characteristics of the recorded signals. For example Molina et al. showed that emission patterns are not random and proposed stochastic [15] and chaos-deterministic approaches [16] to characterize their evolution with time.

Recently, Nakayama et al. [19, 20] and Matta et al. [21] have made significant advances in infrared and ultraviolet imaging of the triboplasma, generated at the rear of sliding contacts by gas discharge under ambient conditions. The research described in the current paper differs from these 
studies, since we are concerned with electron emission mechanisms before interactions with surround gas take place.

One of the main limitations of electron triboemission experiments to date is that they provide only ensemble average values, giving no information relating to spatial location or direction. This shortcoming is, in part, responsible for our lack of understand of triboemission processes, since it prevents emission measurements from being directly correlated with other surface analyses, such as SEM and AFM. To address this issue, we present experiments using a microchannel plate (MCP), in which spatial maps of electron emission are obtained (see Figure 2). The MCP - which is effectively a dense array of electron multipliers - is located within a vacuum chamber above a stationary diamond stylus that is loaded against a rotating aluminium disc covered with a $5 \mu \mathrm{m}$ oxide layer (a brittle material on a soft substrate is likely to form cracks and thus induce emission). In this way, bursts of electrons can be visualised, revealing the pattern, direction, and temporal characteristics of emission.

\section{Apparatus}

The vacuum pin-on-disc tribometer used in this study was based on a high-pressure Elasto-Hydrodynamic rig from PCS Instruments Ltd, (Acton, UK), and is shown schematically in Figure 3. The tribometer consists of a stainless steel chamber with an external motor that drives the disc shaft rotation via a $\mathrm{SiC}$ face seal. A $10^{-5}$ mbar vacuum was obtained in the chamber using a turbomolecular pump from Edwards Vacuum Ltd, connected by a KF25 flange. A wide range pressure gauge measured the pressure inside the chamber.

The sliding contact was produced by loading a $100 \mu \mathrm{m}$ radius diamond tip (manufactured by Synton-MDP Ltd) against the disc specimen by means of a $1 \mathrm{~mm}$ diameter stainless steel arm and a simple dead weight arrangement. Strain gauges have been bonded to the stainless steel arm in order to measure the friction between the tip and the disc.

The MCP is circular with a "Chevron" arrangement (i.e. two MCPs in series for increased sensitivity), manufactured by Photonis Ltd. It has an active diameter of $75 \mathrm{~mm}$ and is located $10 \mathrm{~mm}$ above the disc specimen. The MCP channels have diameters of $10 \mu \mathrm{m}$ and their centre-to-centre distance is $12 \mu \mathrm{m}$. A P20 phosphor screen, also from Photonis Ltd is mounted on top of the MCP to convert electrons from the MCP into photons. The MCP and the phosphor screen are mounted in an 8" CF flange - which also acts as the lid of the chamber sealed using a copper ring. Three high voltage connectors are incorporated in the flange in order to apply voltages to the MCP input and output, and the phosphor screen. Images were acquired using a Phantom Miro ex2 camera with a Fujian 35 $\mathrm{mm} \mathrm{f} 1.7$ lens, focussed on the phosphor screen.

An Aluminium disc, with a $100 \mathrm{~mm}$ diameter and $10 \mathrm{~mm}$ thickness, was used as a sample. This was anodized by Metro Engineering and Plating Works Ltd (Uxbridge UK) to produce a surface oxide layer that was $5 \mu \mathrm{m}$ in thickness. Prior to testing, the chamber was cleaned with toluene and isopropanol, and then vented for 4 hours. The disc sample was also cleaned with toluene and isopropanol, in an ultrasonic bath for 15 minutes each. The diamond tip was then put in contact with the aluminium disc and loaded with a dead weight of $0.4 \mathrm{~N}$ 
Then, the flange was sealed to close the chamber and the pump operated until a vacuum of $10^{-5}$ mbar was reached.

A 2-channel high voltage power supply PF1054 from Photonis Ltd allowed $2 \mathrm{kV}$ to be applied to the MCP output and $5 \mathrm{kV}$ to the phosphor screen input. The camera was focussed on the phosphor screen, capturing $640 \times 480$ images, (with a $217 \mu \mathrm{m} /$ pixel spatial resolution) at a rate of $100 \mathrm{frames} / \mathrm{s}$ (with a shutter speed of $\sim 10 \mathrm{~ms}$ ). A dark sheet is placed over the rig to shield the phosphor screen from light. Finally, the camera and the strain gauge acquisition were started before the disc was rotated for $120 \mathrm{~s}$ so that the linear velocity of the contact was $44 \mathrm{~mm} / \mathrm{s}$.

\section{Results and Discussion}

\subsection{Averaged measurements}

The evolution of the average intensity over the whole phosphor screen, for each frame, as a function of time, is given in Figure 4. Here, an almost constant low emission level is seen, onto which several high bursts of emission are superimposed. As observed by Nakayama et al. [12] and Molina et al. [16] for alumina specimens, electrons are emitted in bursts, shortly after scratching starts. The coefficient of friction throughout this test was measured at 0.1 .

Figure 5 shows the probability distribution of the normalized average intensities from the $10 \mathrm{~ms}$ exposures shown in Figure 4. The probability of occurrence is defined by the number of video frames for which the intensity falls within a particular range, $\left[I_{j} I_{j+1}\right]$, divided by the total number of video frames $N$ :

$$
P\left(I_{j}, I_{j+1}\right)=\frac{N\left(I_{j}, I_{j+1}\right)}{N}
$$

It can be seen that more than $93.5 \%$ of the captured fames have intensities between 0 and $5 \%$ of the maximal intensity. The probability of occurrence decreases with intensity (falling to $0.01 \%$ for the highest range). If the highest bursts of emission are defined as those for which the intensity in the screen is at least $25 \%$ of the maximal value, then 80 such events are detected during the $120 \mathrm{~s}$ test, (i.e. 0.66 events/s or 15 events/m or 3.68 events/ revolution).

The probability distribution can then be used to calculate the spectral entropy (SE) following Molina et al. [16]:

$$
S E\left(I_{0}, I_{M A X}\right)=\sum_{j}\left\{P\left(I_{j}, I_{j+1}\right) \log \left(\frac{1}{P\left(I_{j}, I_{j+1}\right)}\right)\right\}
$$

and from this, the Shannon spectral entropy (SSE) can be calculated:

$$
\operatorname{SSE}\left(I_{0}, I_{M A X}\right)=\frac{S E\left(I_{0}, I_{\text {MAX }}\right)}{\log \left(N Z\left(I_{0}, I_{M A X}\right)\right)}
$$

where $N Z$ is the number of non zero probabilities. The SSE value reflects the complexity of a signal. For example, a white noise signal spectrum is truly random and has a SSE of 1 whereas a simple signal (such as a sum of sinusoids) 
has a $S S E$ close to zero. For the intensity signal displayed above, obtained using a $10 \mathrm{~ms}$ window, a SSE of 0.11 has been found. This suggests that the bursts of emission are not random and can be explained by deterministic mechanisms. Obviously, this value depends on the time window used. For $20 \mathrm{~ms}$ and $40 \mathrm{~ms}$ time windows, SSE values are 0.16 and 0.23 respectively. This is in agreement with Molina et al., who found 0.26 for a diamond tip on alumina contact with a 40 ms time window [16].

In order to locate these emission events on the disc sample, Figure 6 gives a map of the normalized average intensity of the whole phosphor screen as a function of the angular position on the disc and the number of the revolution (the log value is given for a better contrast). During the first six cycles, there is no emission as the disc is not turning. Then events become progressively more frequent. We can see that most of the events occur on a section of the disc specimen $\left(75^{\circ}-275^{\circ}\right)$. This may be due to misalignment or non-planarity of the sample. It is also interesting to note that, at certain positions on the disc, emission occurs at almost every pass. For example, at $20^{\circ}$, a relatively high intensity is detected from cycle 13 onwards. This suggests that emission is not random and is due to a systematic deformation/fracture process. Further inspection of this high intensity line, reveals a slight positive gradient, indicating that the source of emission is progressing slowly around the wear track with each cycle.

Figure 6 also shows weakly visible vertical lines, which may indicate after-emission (i.e. electrons being emitted from the wear track, rather than from the contact). The following section analyses the individual spatial distribution of electrons in order to provide further information on these mechanisms.

\subsection{Spatially resolved measurements}

An example of a snapshot from the phosphor screen, obtained during a high intensity approximately circular burst of emission, is shown in Figure 7. Here, the tip is stationary, with a position, indicated by the red cross, while the disc is turning anticlockwise. It can be seen that electrons are emitted in all directions. Several of them even hit the MCP at a lateral distance of $60 \mathrm{~mm}$ from the contact, indicating an angle of emission less than $10^{\circ}$ to the sample surface. However, the majority of electrons are emitted towards the front of the contact and towards the centre of the disc. A maximum density is found close to the inlet of the contact. This is contrary to standard conceptual representation of triboemission [22] where the emission is shown to come from the outlet. This discrepancy is probably due to the standard representation being based on experiments in atmosphere where a significant portion of emission arises due to gaseous discharge on the freshly worn surface at the rear of the contact [19]. In our case, the emission is likely to come exclusively from the fractures in the sample. The stronger emission at the inlet can be explained by the ploughing of the surface material during abrasion.

Figure 8 shows a normalized average of the 80 emission maps of maximal intensity. This shows more clearly that the maximum emission is located at the inlet of the contact. The low emission towards the exterior of the disc may be due to a partial obstruction of the electrons by the tip itself. This is supported by the presence of a region of reduced emission at the location of the scratching tip. 
The distribution and quantity of emitted electrons varied considerably between each burst, with the most frequently observed pattern being that shown in Figure 6. However, two other types of emission patterns were also been observed repeatedly. These consist of remarkably straight lines radiating out from the contact in one of two directions, in either a South-West (Figure 9a) or a South-East (Figure 9b) direction. It is suggested that these emission lines, which are far less frequent than the type of patterns of Figure 3a, are caused by cracks occurring in or along the wear track during abrasion. The average angle with the wear track has been found around $60^{\circ}$ with a standard deviation of 12 . This experimental evidence supports the hypothesis that triboemission occurs due to charge separation resulting from crack formation [18]. This technique of triboemission visualisation by an MCP could provide a powerful tool to visualize crack growth, in real time, giving information on their size and direction. One of the strengths of this technique is that the microscopic deformation events that lead to emission are magnified greatly when viewed on the phosphor screen (see Figure 2). Because of the rough nature of the $\mathrm{Al}_{2} \mathrm{O}_{3}$ it was not possible to measure corresponding defects on the worn surface in this study. However, further investigation is planned, in which emission distributions are compared with surface analysis measurements, using techniques such as scanning electron microscopy, in order to relate characteristics of the emission pattern to cracks width and depth.

Figure 6 showed that, at some specific positions on the disc sample, emission systematically occurs. In order to question the randomness of the emission leading to the patterns presented in this section (South-West lines, South-East lines, and high intensity distributions), a polar plot is given in Figure 10 where these events are shown at the disc position where they occurred. In this plot, the angle $\left({ }^{\circ}\right)$ defines the angular position on the disc and the radius is the number of the revolution. A few events attributed to cracks are isolated but several others clearly repeat at several passings (such as at $20^{\circ}$ or $85^{\circ}$ ). This suggests that the same created (or pre-existing) crack is being deformed at each pass.

In most triboemission studies found in the literature, emission ceases as soon as the scratching stops [12]. For instance, Molina et al. observed this for semi-conductors, though they mentioned that significant post-contact emission occurred for insulators [23]. During our sliding tests, a high burst of emission is generally not followed by emission in the subsequent video frames. However, occasionally we observe a burst of emission at the scratching tip, followed by a pattern of emission that rotates on the screen with the same angular velocity as the disc - i.e. electrons are being emitted from a single location on the wear track after contact has occurred. An example of this behaviour is highlighted in Figure 11 where several snapshots of the phosphor screen are shown, during and after an initial burst of emission at the tip. Here, emission from the wear track continues more than one second after the initial burst. This prolonged emission (which fluctuates over time) is unlikely to be due to a crack propagating and may be a consequence of charging of the surface. Our technique, because of its large field of view, is therefore able to distinguish between emission from the contact and after-emission from the whole wear track.

These results suggest that triboemission from the $\mathrm{Al}_{2} \mathrm{O}_{3}$ surface occurs due to at least three mechanisms: $i$ ) that shown in Figure 7, which may be due to 
deformation of the surface in front of the sliding tip ii) that shown in Figure 9, which is attributed to crack formation, iii) that shown in Figure 11, being emitted from the wear scar after contact has occurred. This combination of events some occurring at certain disc positions and some occurring more randomly - is likely to explain the semi random nature of the spectral entropy observed by us and Molina et al.

\section{Conclusion}

We report on experiments carried out on a new vacuum tribometer using MCPs. This has enabled us, for the first time, to map the emission of electrons occurring in bursts during scratching tests. The patterns obtained show different types of emission. The most common distribution is approximately circular in shape with a maximum intensity close the front of the contact. Straight lines have also been observed and are attributed to crack growth. Sporadic post contact emission from the wear track has been detected as well. These experiments pave the way for further investigations in which these triboemission measurements are correlated with other surface analysis techniques in order to further elucidate emission mechanisms. This should aid our understanding of boundary film formation and lubricant degradation. The technique may also be useful as an in-situ tool for monitoring cracks formation and growth.

\section{Acknowledgements}

This work was funded through the European Metrology Research Programme (EMRP) Project IND11 MADES. The EMRP is jointly funded by the EMRP participating countries within EURAMET and the European Union.

The authors are also very grateful to Dr Clive Hamer of PCS Instruments for providing the custom-built tribometer adapted in this work and to Professor Iain Baikie of KP Technology (Wick, UK) for insightful discussions.

\section{References}

[1] Kajdas, C., Furey, M. J., Ritter, A. L., Molina, G. J.: Triboemission as a basic part of the boundary friction regime. Lubr. Sci. 14, 223-254 (2002)

[2] Baxter, W. J.: A study of plastic deformation by exoelectron emission. Vacuum 22, 571-575 (1972)

[3] Becker, K.: Stimulated exoelectron emission from the surface of insulating solids. Crit. Rev. Solid State Sci. 3, 39-81 (1972)

[4] Furey, M. J., Kajdas, C., Kempinski, R., Tripathy, B.: Action mechanism of selected vinyl monomers under boundary lubrication of an alumina-onalumina system. Lubr. Sci. 10, 3-25 (1997)

[5] Furey, M. J., Kajdas, C.: Tribopolymerization as a Mechanism of Boundary Lubrication. Surface Modification and Mechanisms, Chap. 7, Totten G. E. and Liang H., eds. Marcel-Dekker, New York, pp. 165-201 (2004).

[6] Spikes, H. A.: The history and mechanisms of ZDDP. Tribol. Lett. 17, 469-489, (2004) 
[7] Zhoa, X., Bhushan, B.: Studies on Degradation Mechanisms of. Lubricants for Magnetic Thin-Film Rigid Disks. Proc. Inst. Mech. Eng., Part J: J. Eng. Tribol. 215, 173-188 (2001)

[8] Ishihara, T., Tanaka, K., Fujita, K., Hirao, K., Soga, N.: Full Color Triboluminescence of Rare-Earth-Doped Hexacelsian $\left(\mathrm{BaAl}_{2} \mathrm{Si}_{2} \mathrm{O}_{8}\right)$. Solid State Commun. 107, 763-767 (1998)

[9] James, M. R., Wilson, L., Lane, S. J., Gilbert, J. S., Mather, T. A., Harrison, R. G., Martin, R. S.: Electrical charging of volcanic plumes, Space Sci. Rev. 137, 399418 (2008)

[10] Grunberg, L.: A survey of exo-electron emission phenomena. Br. J. Appl. Phys. 9, 85-93 (1958)

[11] Nakayama, K., Hashimoto, H.: Triboemission from various materials in atmosphere. Wear 147, 335-343 (1991)

[12] Nakayama, K., Suzuki, N., Hashimoto, H.: Triboemission of charged particles and photons from solid surfaces during frictional damage. J. Phys. D: Appl. Phys 25, 303-308 (1992)

[13] Kim, M., Langford, S. C., Dickinson, J. T.: Electron and Photon Emission Accompanying the Abrasion of MgO with Diamond. Tribol. Lett. 1, 147-157 (1995)

[14] Molina, G. J., Furey, M. J., Ritter, A. L., Kajdas, C.: Triboemission from alumina, single crystal sapphire, and aluminium. Wear 249, 214-219 (2001)

[15] Molina, G. J., Furey, M. J., Ritter, A. L., Kajdas, C.: Frequency analysis and modeling of charged-particle triboemission from ceramics. Wear 255, 686694 (2003)

[16] Molina, G. J., Furey, M. J., Kajdas, C.: A deterministic-chaos study of electron triboemission outputs. J. Tribol. 129, 679-683 (2007)

[17] Nakayama, K., Bou-Said, B., Ikeda, H.: Tribo-electromagnetic phenomena of hydrogenated carbon films. Tribo-electrons, -ions, -photons and -charging. Trans. ASME, J. Tribol. 119, 764-768 (1997)

[18] Dickinson, J. T., Jensen, L. C., Jahan-Latibari, A.: Fracto-emission: the role of charge separation. J. Vac. Sci. Technol. A A2, 1112-1116 (1983)

[19] Nakayama, K., Nevshupa, R. A.: Plasma generation in a gap around a sliding contact. J. Phys. D: Appl. Phys. 35, L53-56 (2002)

[20] Nakayama, K.: Triboplasma Generation and Triboluminescence: Influence of Stationary Sliding Partner. Tribol. Lett. 37, 215-228 (2010)

[21] Matta, C., Eryilmaz, O. L., De Barros Bouchet, M. I., Erdemir, A., Martin, J. M., Nakayama, K.: On the possible role of triboplasma in friction and wear of diamond-like carbon films in hydrogen-containing environments. J. Phys D.: Appl. Phys. 42, 075307 (2009)

[22] Nakayama, K.: Triboemission from wearing solid surfaces, ITC Proceedings, Satellite Forum on Tribochemistry, October 28, Tokyo, Japan (1995)

[23] Molina, G. J., Mazilu, D. A, Furey, M. J., Kajdas, C.: On Tribo-emission From the Sliding Contact of Si and Ge. The Annals of University "Dunarea De Jos" of Galat i, Romania, VIII (Tribology), pp. 22-29 (2004) 


\section{FIGURES}

Fig. 1: Schematic illustration of the mechanism of crack-induced tribo-emission

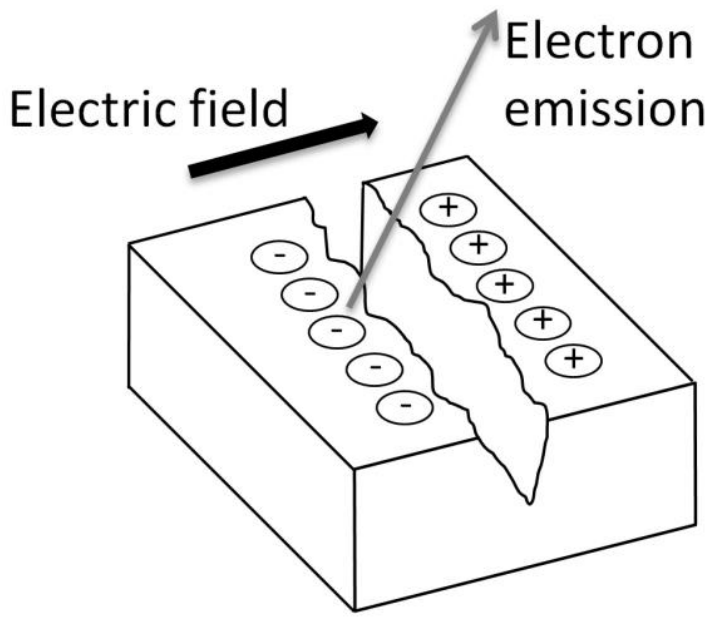


Fig. 2: Schematic illustration of triboemission measurement with a MicroChannel Plate (MCP).

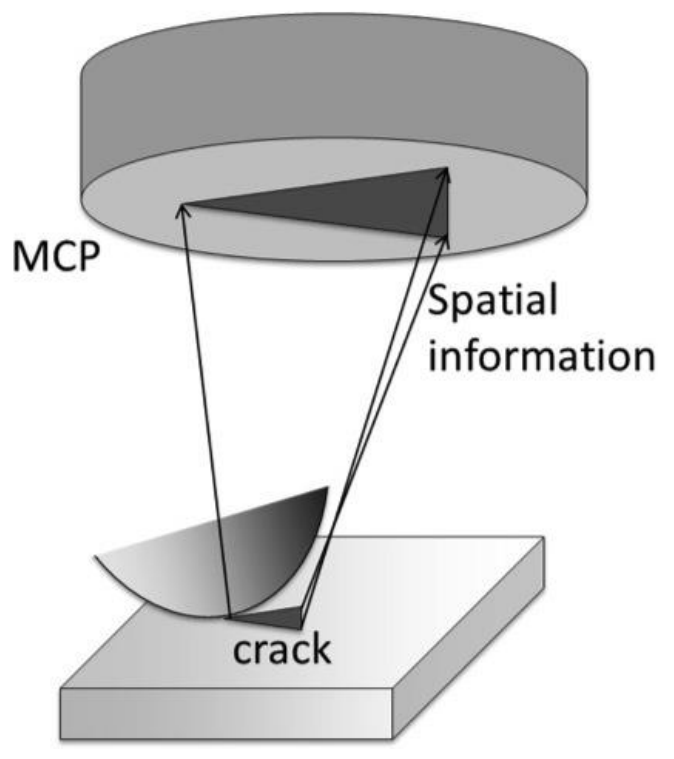


Fig. 3: Schematic diagram of the triboemission rig

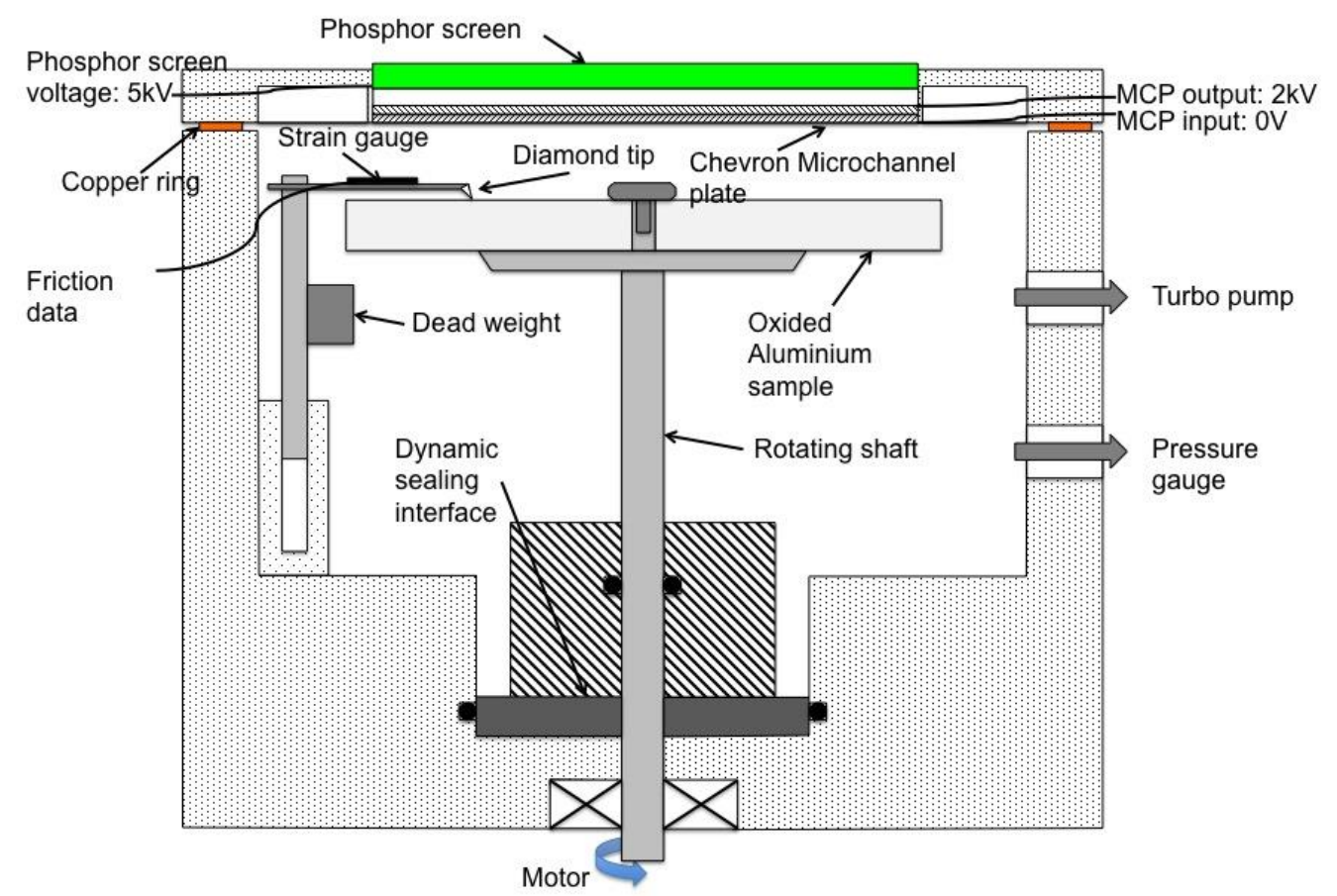


Fig. 4: Normalised value of emission as a function of time

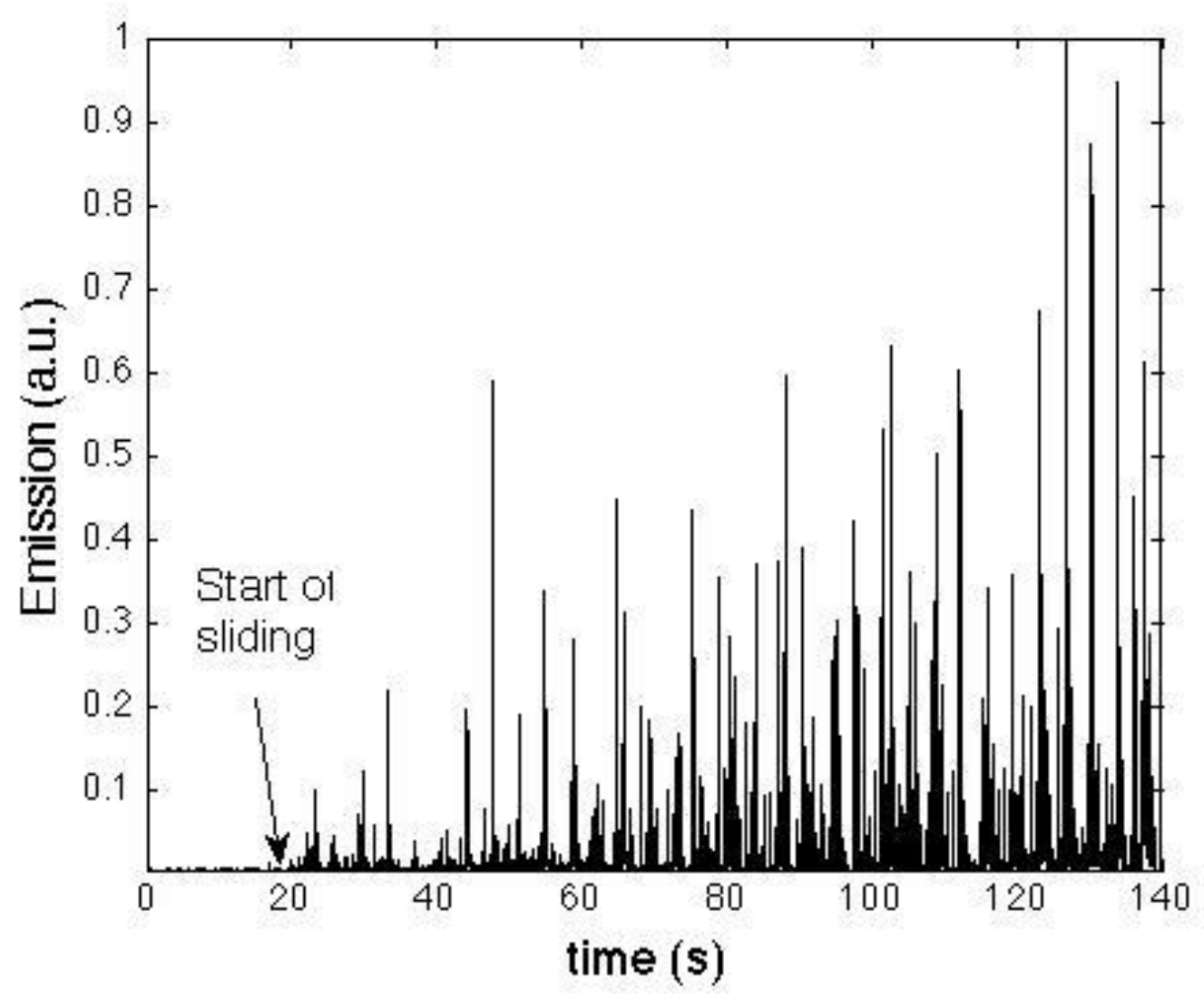


Fig. 5: Probability density of normalized emission levels in the 10 ms window

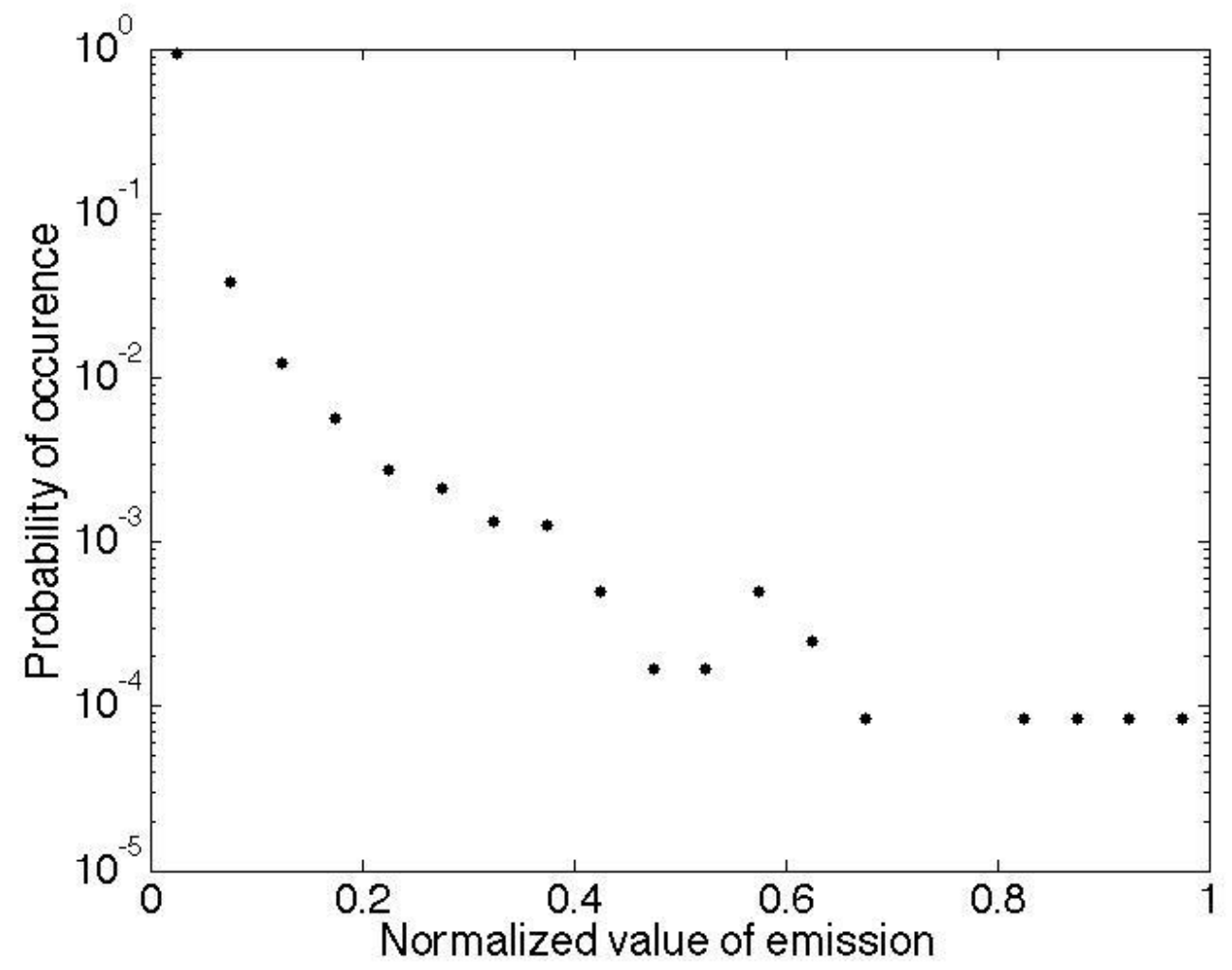


Fig. 6: Intensity in the whole screen as a function of the angular position on the disc sample $\left({ }^{\circ}\right)$ and the number of the revolution.

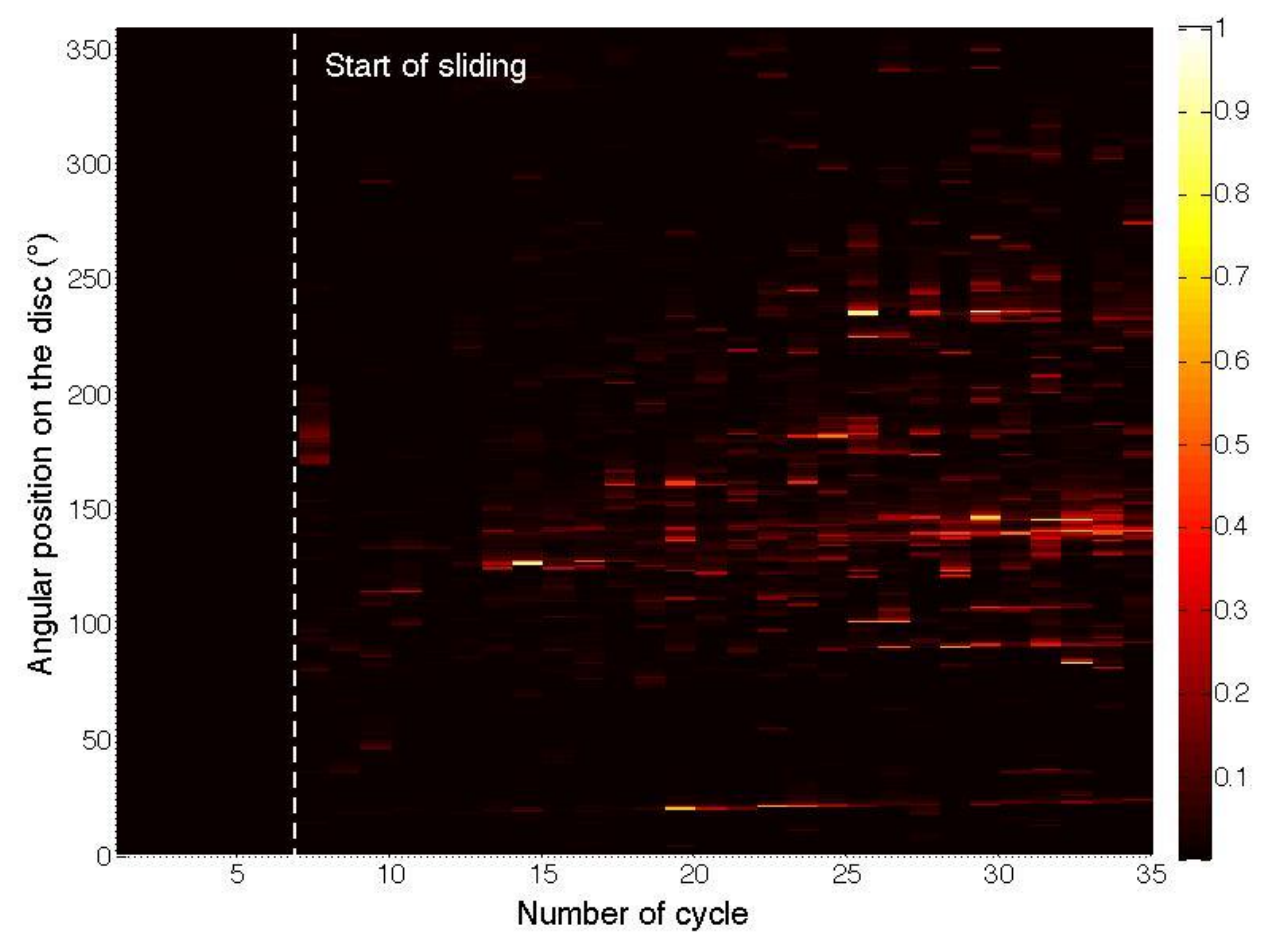


Fig. 7: Phosphor screen snapshot during one burst of emission from the disc specimen. The tip is static and the disc is turning anticlockwise. The red cross indicates the position of the tip.

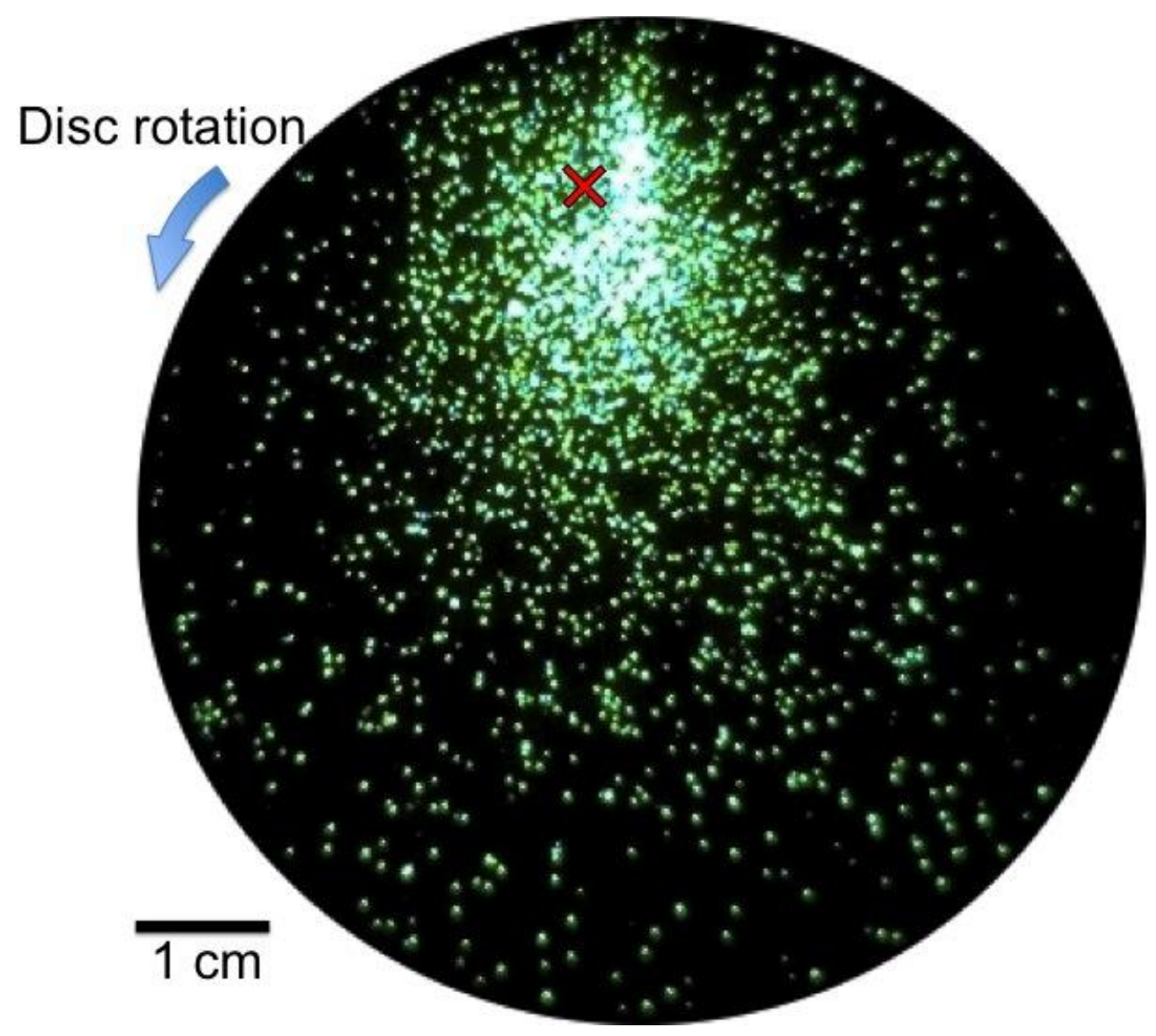


Fig. 8: Normalized maps sum of the 80 most intense bursts of emission

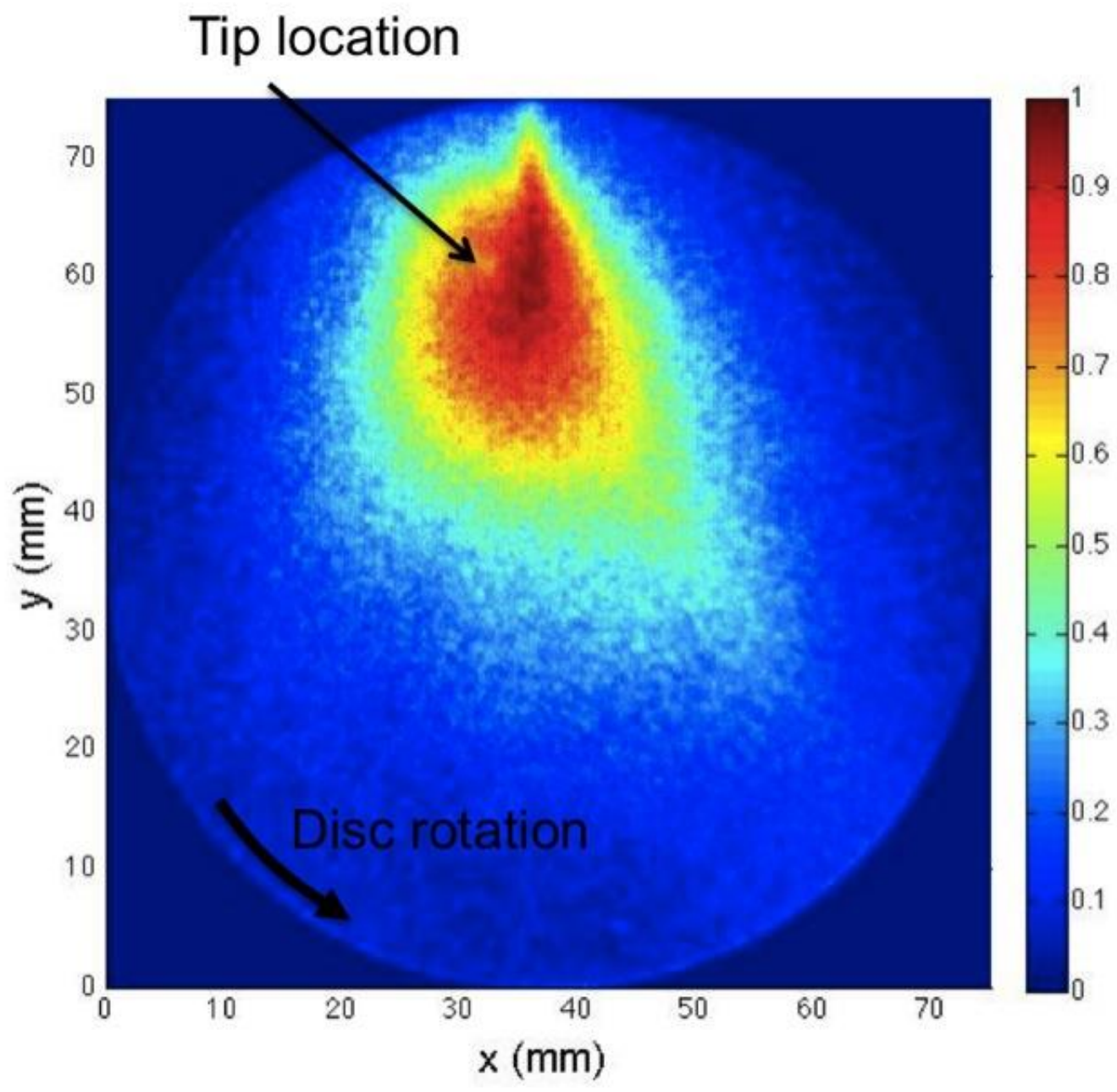


Fig. 9: Snapshots of straight lines radiating from the contact position

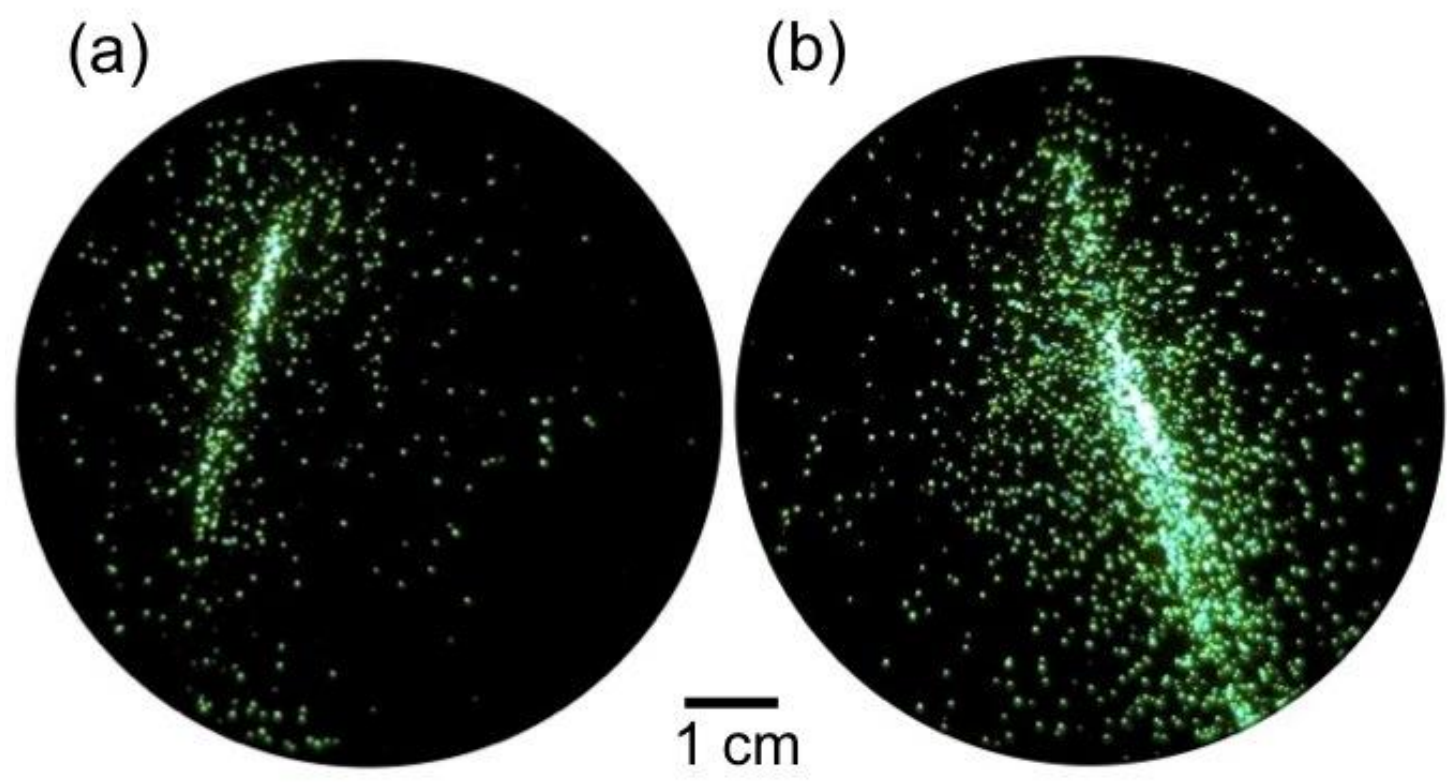


Fig. 10: Polar plot of the locations of left cracks, right cracks and high events. The angle is the angular position on the disc specimen $\left(^{\circ}\right)$ and the radius is the number of the revolution.

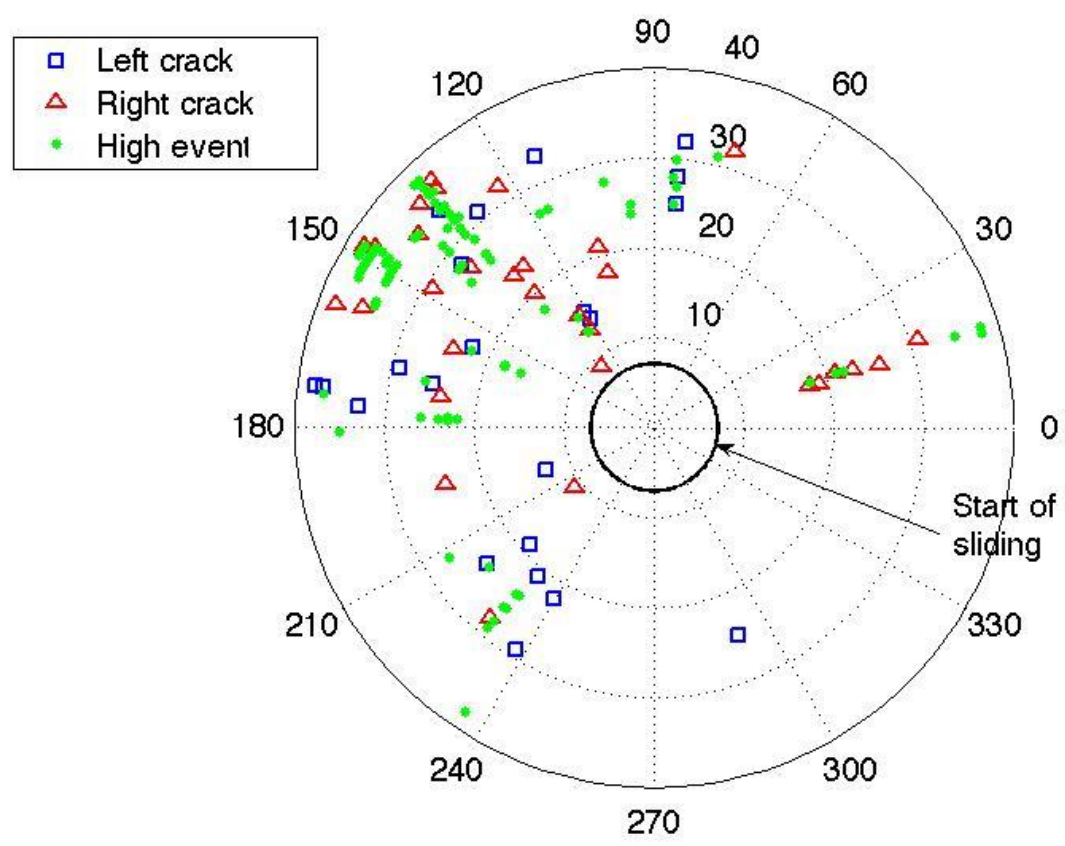


Fig. 11: Several snapshots showing post contact emission of the wear track
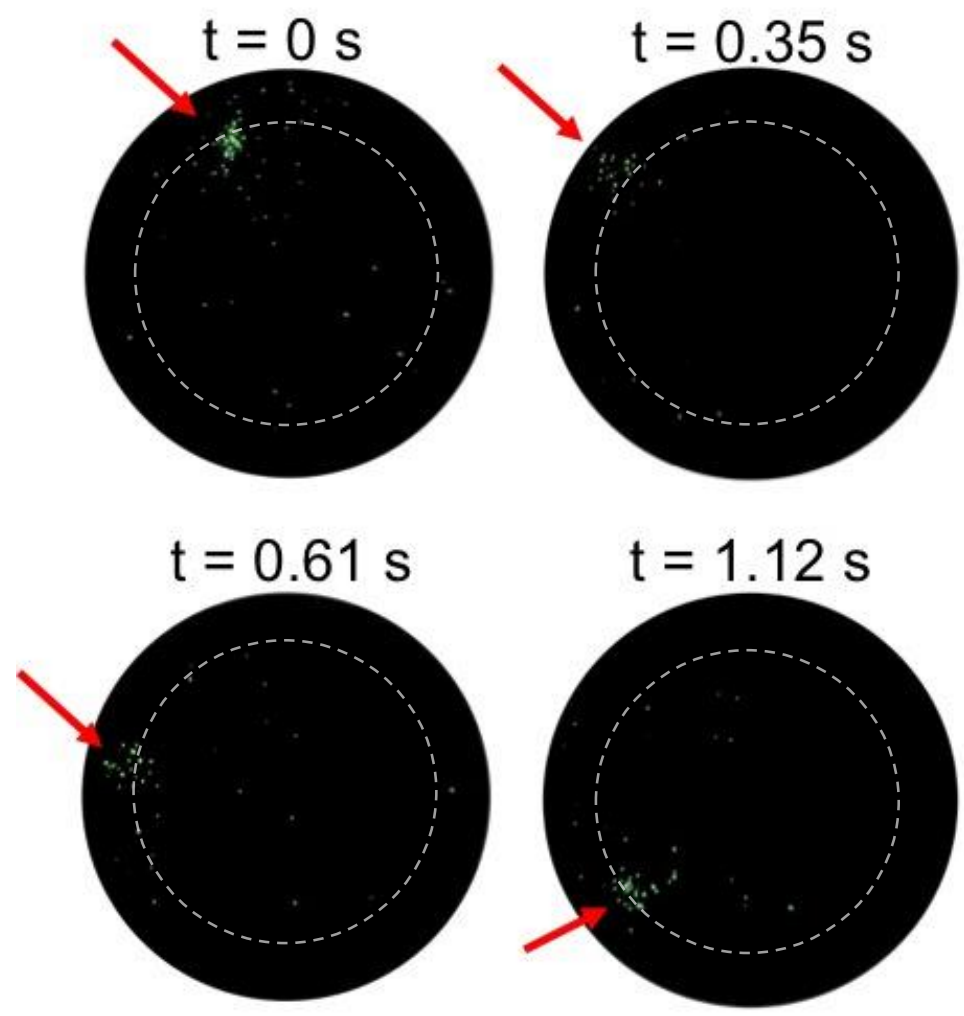